\title{
GEOMETRIC ASPECTS OF THE HOLOGRAPHIC DUALITY
}

\author{
(C) D. V. Bykov*
}

We briefly survey results related to applying the AdS/CFT correspondence to $\mathcal{N}=1$ supersymmetric models. These models, on one hand, are closest to realistic models of elementary particle physics and, on the other hand, are amenable to quantitative analysis using the AdS/CFT correspondence. Furthermore, they are related to such remarkable geometric objects as Sasakian manifolds and Ricci-flat cones, on which we particularly focus.

Keywords: AdS/CFT correspondence, supersymmetry, Sasakian manifold, del Pezzo surface

Dedicated to Andrei Alekseevich Slavnov in honor of his birthday

\section{The AdS/CFT correspondence}

The AdS/CFT conjecture states that particular conformal field theories (CFTs) are in a certain way dual to string theory models describing string propagation in the anti-de Sitter (AdS) space. The original example is the duality between the maximally supersymmetric Yang-Mills theory ( $\mathcal{N}=4$ SYM) and the type IIB superstring in the space $\mathrm{AdS}_{5} \times S^{5}$ [1]. We consider the Yang-Mills theory with the gauge group $S U(N)$ and coupling constant $g_{\mathrm{YM}}$. From the standpoint of the $\mathrm{AdS}_{5} \times S^{5}$ geometry, these two parameters, $N$ and $g_{\mathrm{YM}}$, are related to the radius of the sphere and the flux of the self-dual 5 -form through it: ${ }^{1}$

$$
R^{2} \sim \sqrt{g_{\mathrm{YM}}^{2} N}, \quad \int_{S^{5}} F_{5} \sim N
$$

The correspondence between the two theories is manifested, in particular, in the fact that the global symmetries of field theory correspond to the (super)isometries of $\mathrm{AdS}_{5} \times S^{5}$. Indeed, the superconformal group of the $\mathcal{N}=4$ theory is $P S U(2,2 \mid 4)$. Its maximal bosonic subgroup is $S U(2,2) \times S U(4)$. The first factor here is isomorphic to $S O(2,4)$ (the conformal group of the four-dimensional Minkowski space), and the second factor represents the $R$-symmetry group of the theory. On the other hand, $S O(2,4)$ and $S O(6)$ are the respective isometry groups of the AdS space and the five-dimensional sphere.

The simplest modification of this basic model is obtained if a quotient of the sphere by a discrete subgroup of the isometry group $S O(6)$ is taken:

$$
\mathrm{AdS}_{5} \times S^{5} \longrightarrow \mathrm{AdS}_{5} \times S^{5} / \Gamma, \quad \Gamma \subset S O(6)
$$

The dual gauge theory is now different: the gauge group consists of several simple factors, and the matter multiplets are in bifundamental representations. The number of supersymmetries is also reduced and

*Steklov Mathematical Institute, RAS, Moscow, Russia; Max-Planck-Institut für Gravitationsphysik, AlbertEinstein-Institut, Potsdam-Golm, Germany, e-mail: dbykov@mi.ras.ru,dmitri.bykov@aei.mpg.de.

${ }^{1}$ We note that the form $F_{5}$ also has nonzero components along the AdS space because it is self-dual.

Translated from Teoreticheskaya i Matematicheskaya Fizika, Vol. 181, No. 3, pp. 436-448, December, 2014. Original article submitted June 24, 2014. 
depends on the structure of the group $\Gamma$ : if $\Gamma \subset S U(3) \subset S O(6)$, then one supersymmetry, $\mathcal{N}=1$, remains. But if $\Gamma \subset S U(2) \subset S U(3) \subset S O(6)$, then we obtain an $\mathcal{N}=2$ superconformal field theory. Discrete subgroups of $S U(2)$ were classified by Klein and are known as the Kleinian groups $A_{n}, D_{n}$, and $E_{6,7,8}$. Hypermultiplets in this theory can be obtained by taking a quotient from the set of fields of the theory with $\Gamma=1$ (i.e., from the $\mathcal{N}=4 \mathrm{SYM}$ ) [2]. It turns out that they are described by the so-called quiver, corresponding to the Dynkin diagram of the Lie algebra of the type $A-D-E[3]$.

We note that the quotient manifold contains singularities if the action of the group $\Gamma$ on $S^{5}$ is not free. This is precisely what happens if $\Gamma \subset S U(2)$. This means that there should exist a more general smooth metric containing certain "singularity-resolution" parameters and becoming nonsmooth in the limit as these parameters tend to zero.

\section{Sasakian manifolds and $\mathcal{N}=1$ supersymmetry}

All manifolds of the type $\mathrm{AdS}_{5} \times S^{5} / \Gamma$ mentioned in the preceding section are particular cases of a rather wide class of solutions of IIB supergravity of the form $\mathrm{AdS}_{5} \times X^{5}$, in which the dilaton $\phi$ is constant and the only nonzero form is the 5 -form:

$$
\phi=\text { const }, \quad F_{5} \sim N\left((\operatorname{vol})_{\mathrm{AdS}_{5}}+(\operatorname{vol})_{X^{5}}\right) .
$$

The Bogomolnyi-Prasad-Sommerfeld condition for this configuration, i.e., the requirement that at least one supersymmetry be preserved, reduces to the gravitino variation vanishing (we write the variation schematically; see, e.g., [4] for more details):

$$
\delta \psi_{\mu}=\left(\nabla_{\mu}+F_{5} \gamma_{\mu}\right) \epsilon=0
$$

The dilatino variation is automatically zero for the considered configuration. The spinor $\epsilon$ satisfying (2) is called a Killing spinor. The existence of a Killing spinor substantially constrains the geometry of the space $X^{5}$. In particular, similarly to how this was done in [5], it can be shown that the solubility of Eq. (2) is equivalent to the requirement that the metric cone over $X^{5}$ be Kähler and Ricci-flat. In other words, let $\left(\widetilde{d s^{2}}\right)_{X^{5}}$ be the metric on $X^{5}$. The metric on the cone is $d s^{2}=d r^{2}+r^{2}\left(\widetilde{d s^{2}}\right)_{X^{5}}$ by definition. The supersymmetry requirement is that the metric $d s^{2}$ be Kähler and Ricci-flat (the latter also follows directly because $\left(\widetilde{d s^{2}}\right)_{X^{5}}$ is an Eintein metric of positive curvature by the supergravity equations of motion and 5 -form (1)). In this case, the metric on the base of the cone $\left(\widetilde{d s^{2}}\right)_{X^{5}}$ is said to be Sasaki-Einstein.

All Sasaki-Einstein metrics look rather simple locally: they can be written in the form

$$
\left(d s^{2}\right)_{X^{5}}=(d \varphi-A)^{2}+\sum_{i, j=1}^{2} g_{i \bar{j}} d z_{i} d \bar{z}_{j},
$$

where $g_{i \bar{j}}$ is a Kähler-Einstein metric on some complex surface (the meaning of this surface is explained in Sec. 2.2.1 below) and $A$ is the Kähler current. The validity of such a representation is clear for the sphere $S^{5}$ if we recall that it is the total space of a Hopf bundle with the base $\mathbb{C P}^{2}$. An important difference in the general case is that $g_{i \bar{j}}$ need not necessarily be smooth (although the metric on $X^{5}$ is smooth).

On a Sasaki-Einstein manifold with metric (3), there is a canonical vector field $\partial / \partial \varphi$ with a fixed norm, the so-called Reeb vector. From the standpoint of the dual gauge theory, this $U(1)$ isometry of $X^{5}$ is dual to the global $U(1)$ symmetry of the superconformal field theory, the $R$-symmetry, which acts on the supercharges [6]:

$$
Q \rightarrow e^{i \alpha} Q, \quad \bar{Q} \rightarrow e^{-i \alpha} \bar{Q} .
$$

In contrast to an arbitrary theory with the $\mathcal{N}=1$ supersymmetry, a superconformal theory always has a $U(1) R$-symmetry because the generator of the $R$-symmetry enters the superalgebra $S U(2,2 \mid 1)$ explicitly (the $R$-transformations are therefore its internal automorphisms). 
2.1. Calabi-Yau manifolds and singularities. The construction of a cone over a Sasaki-Einstein manifold $X^{5}$, described above, allows taking a new look at the configuration of the type $\operatorname{AdS}_{5} \times X^{5}$. Here, we can recall the interpretation of the $\mathcal{N}=4$ SYM theory as an effective field theory describing oscillations of a stack of parallel D3 branes embedded in the flat space $\mathbb{R}^{1,9}$. Clearly, the space transverse to the branes in this case is $\mathbb{R}^{6}$. The branes are massive objects and therefore change the geometry of the space in which they are embedded. Assuming that the branes are located at the origin of $\mathbb{R}^{6}$, we can introduce a radial coordinate $r$ and seek supergravity solutions of the type

$$
d s^{2}=h^{-1 / 2}(r) \sum_{i=1}^{4} d x_{i}^{2}+h^{1 / 2}(r)\left(d r^{2}+r^{2}(d \Omega)_{S^{5}}\right) .
$$

Because the branes are charged with respect to the 5 -form $F_{5}$, we must also impose the condition $\int_{S^{5}} F_{5} \sim N$. The solution has the form [7] ( $l$ is a linear scale)

$$
h(r)=1+\frac{l^{4} N}{r^{4}} .
$$

In the limit $r \rightarrow 0$, we obtain the metric of $\mathrm{AdS}_{5} \times S^{5}$. It is hence clear that the sphere $S^{5}$ emerges as the locus of points equidistant from $r=0$ (in sense of the natural flat metric) in the six-dimensional space in which the branes are embedded. We can now consider the case where the "internal" six-dimensional space is not a flat space but an arbitrary (compact) Calabi-Yau space $Y^{6}$. Placing the branes at a nonsingular point of $Y^{6}$, we again have the $\mathcal{N}=4 \mathrm{SYM}$ as a low-energy limit. But the situation changes if we place the branes at a singular point of the Calabi-Yau space. In this case, the effective field theory on the branes depends on the local geometry of $Y^{6}$ in the vicinity of the singularity. The neighborhood of the singularity can be described by a noncompact Ricci-flat metric of the conical type, i.e., a metric of the form $\left(d s^{2}\right)_{\text {sing }}=d r^{2}+r^{2}\left(\widetilde{d s^{2}}\right)_{X^{5}}$. In this case, the points equidistant from the singularity form not an $S^{5}$ but a Sasaki-Einstein manifold $X^{5}$. Ansatz (4) still holds and leads to the configuration $\operatorname{AdS}_{5} \times X^{5}$ in the limit $r \rightarrow 0[8]$.

2.1.1. The cones. It follows from the preceding section that the spaces $X^{5}$ are tightly connected with the singularities of the Calabi-Yau manifolds $Y^{6}$ of complex dimension three. Some of these singularities can be represented as singularities of complex cones over complex surfaces, hereafter denoted by $\mathcal{M}$. We explain what this means with the example of the so-called "conifold." We consider a nondegenerate quadric in $\mathbb{C P}^{3}$, for instance,

$$
X_{0}^{2}+X_{1}^{2}+X_{2}^{2}+X_{3}^{2}=0
$$

It is known that this algebraic variety is isomorphic to $\mathbb{C P}^{1} \times \mathbb{C P}^{1}$. It is nonsingular as a hypersurface in $\mathbb{C P}^{3}$. To pass to the cone, we "forget" that $X_{0}, X_{1}, X_{2}$, and $X_{3}$ are projective coordinates, i.e., we consider them ordinary affine variables. In other words, we consider Eq. (6) in $\mathbb{C}^{4}$. Obviously, singularity at the origin $X_{0}=X_{1}=X_{2}=X_{3}=0$ then arises. We call this singular variety the complex cone over $\mathcal{M}=\mathbb{C P}^{1} \times \mathbb{C P}^{1}$. This definition can be obviously extended to complex cones over other complex surfaces.

The only remaining question is in which case the affine manifolds of sort (6) are Ricci-flat (or, more precisely, in which case they remain Ricci-flat after the singularity at the origin has been resolved). It turns out that this requirement is met if and only if the complex surface $\mathcal{M}$ has an ample anticanonical bundle. In the language of differential geometry, this means that $\mathcal{M}$ is a positively curved surface or, more exactly, that the integral of the Ricci form over any homologically nontrivial two-cycle $C$ is positive:

$$
\int_{C} \frac{i}{2 \pi} R_{m \bar{n}} d z_{m} \wedge d \bar{z}_{n}>0
$$


It is known from algebraic geometry that the only compact nonsingular surfaces of positive curvature are $\mathbb{C P}^{1} \times \mathbb{C P}^{1}, \mathbb{C P}^{2}$, and also the blow-ups of $\mathbb{C P}^{2}$ in no more than eight points. We let $\mathbf{d} \mathbf{P}_{1}, \ldots, \mathbf{d} \mathbf{P}_{8}$ denote the surfaces arising from such blow-ups; these are the so-called del Pezzo surfaces (two-dimensional Fano varieties).

We choose one of these surfaces $\mathcal{M}$. How can a corresponding Sasaki-Einstein metric be constructed on $X^{5}$ ? If $\mathcal{M}$ admits a Kähler-Einstein metric (with a Kähler potential $K$ ), then the answer is given by formula (3). It can be also rewritten differently if we recall the correspondence between the Kähler-Einstein metrics on $\mathcal{M}$ and Ricci-flat metrics on $Y^{6}$. We can seek a Kähler potential $\mathcal{K}$ defining the (Ricci-flat) metric on the complex cone over $\mathcal{M}$ in the form

$$
\mathcal{K}=\mathcal{K}\left(|u|^{2} e^{K}\right)
$$

This ansatz is called the Calabi ansatz [9].

2.1.2. The $\mathbb{C}^{3} / \mathbb{Z}_{3}$ singularity. We consider the situation where the Calabi ansatz is applicable, namely, a singularity of the type $\mathbb{C}^{3} / \mathbb{Z}_{3}$, where $\mathbb{Z}_{3}$ acts on $\mathbb{C}^{3}$ via multiplication by a root of unity:

$$
\left(z_{1}, z_{2}, z_{3}\right) \rightarrow e^{2 \pi i / 3}\left(z_{1}, z_{2}, z_{3}\right) .
$$

The minimum set of invariant coordinates is given by all possible cubic combinations of the original variables $z_{1}, z_{2}$, and $z_{3}$. Clearly, these combinations are no longer independent: they satisfy certain equations. If the variables $z_{1}, z_{2}$, and $z_{3}$ were projective, then these cubic combinations would provide the linear system $|\mathcal{O}(3)|$ of sections of the anticanonical bundle $\mathcal{O}(3)$ over $\mathbb{C P}^{2}$. Because the variables $z_{1}, z_{2}$, and $z_{3}$ are in fact affine, the resulting manifold is the total space of the canonical bundle $\mathcal{O}(-3)$ over $\mathbb{C P}^{2}$ or, in the previously introduced terminology, it is the cone over $\mathcal{M}=\mathbb{C P}^{2}$.

The Kähler potential of $\mathbb{C P}^{2}$ is written in the inhomogeneous coordinates as

$$
K=\log \left(1+\left|w_{1}\right|^{2}+\left|w_{2}\right|^{2}\right),
$$

and Calabi ansatz (7) in this case can therefore be brought to the form $\mathcal{K}=\mathcal{K}\left(\left|z_{1}\right|^{2}+\left|z_{2}\right|^{2}+\left|z_{3}\right|^{2}\right)$ by a simple change of variables. The Ricci-flatness equation is an ordinary differential equation for $\mathcal{K}$, which can be solved explicitly (see [10] for more details). At infinity, i.e., when $\left|z_{1}\right|^{2}+\left|z_{2}\right|^{2}+\left|z_{3}\right|^{2} \rightarrow \infty$, the metric becomes asymptotically Euclidean, but the requirement that there should be no conical defect at the origin leads to the necessity of taking the quotient by (8). Hence, the metric at infinity asymptotically approaches the metric on $\mathbb{C}^{3} / \mathbb{Z}_{3}$, while the singularity at the origin is resolved using the blow-up of a copy of $\mathbb{C P}^{2}$. Writing the metric on $\mathbb{C}^{3} / \mathbb{Z}_{3}$ in conical form, we see that the corresponding Sasaki-Einstein manifold is the (smooth) lens space $S^{5} / \mathbb{Z}_{3}$.

2.1.3. The conifold. Apart from sphere quotients, the most studied Sasaki-Einstein manifold is related to the conifold defined by algebraic equation (6). A Ricci-flat metric on conifold (6) was obtained in [11] and later generalized in [12]. This metric can be constructed using the substitution

$$
\mathcal{K}=a \log \left(1+|u|^{2}\right)+K\left(\left(1+|u|^{2}\right)\left(\left|w_{1}\right|^{2}+\left|w_{2}\right|^{2}\right)\right) .
$$

The Ricci-flatness equation can once again be solved explicitly. At "infinity," the metric is expressible as a cone over a Sasaki-Einstein manifold, which in this case is called

$$
T^{11}:=\frac{S U(2) \times S U(2)}{U(1)}
$$


(the group $U(1)$ is embedded diagonally). The dual $\mathcal{N}=1$ field theory was introduced in [13]. It is a gauge theory with the gauge group $S U(N) \times S U(N)$ and two sets of chiral fields $A_{i}$ and $B_{j}, i, j=1,2$, which form doublets with respect to the two global $S U(2)$ groups. The chiral fields $A_{i}$ and $B_{j}$ are in bifundamental representations $(N, \bar{N})$ and $(\bar{N}, N)$, and the superpotential is given by

$$
W=\epsilon^{i j} \epsilon^{m n} \operatorname{tr}\left(A_{i} B_{m} A_{j} B_{n}\right)
$$

2.1.4. Del Pezzo surfaces. We note that not all positively curved surfaces admit Kähler-Einstein metrics. More precisely, Kähler-Einstein metrics do not exist on the del Pezzo surfaces $\mathbf{d} \mathbf{P}_{1}$ and $\mathbf{d P}_{2}$ (see [14]). These surfaces are toric, which means that their isometry group is at least $U(1)^{2}$. We can therefore construct ansatzes for Kähler potentials defining the metrics on cones over these surfaces of the form $K=K\left(\left|u_{1}\right|^{2},\left|u_{2}\right|^{2},\left|u_{3}\right|^{2}\right)$. The Ricci-flatness condition reduces to a Monge-Ampere equation, which can be analyzed in certain cases, for example, for the $\mathbf{d P}_{1}$ surface. In this case, the equation can be solved exactly at "infinity," and the corresponding Sasaki-Einstein metric can be found explicitly. This metric corresponds to the manifold $Y^{2,1}$, a particular case of the $Y^{p, q}$ manifolds first studied in [15]. The del Pezzo surfaces $\mathbf{d} \mathbf{P}_{4,5,6,7,8}$ are not toric but do admit Kähler-Einstein metrics [16], although these are not known explicitly.

We see that the geometry of cones over del Pezzo surfaces has not been fully elucidated. Nevertheless, there exist conjectures for all cases as to what the field theories dual to these supergravity solutions should be. These models were built in [17] (for the toric del Pezzo surfaces) and also in [18] (for the even more general toric Sasakian manifolds $L^{p, q, r}$ obtained in [19]). Dual $\mathcal{N}=1$ field theories for the nontoric del Pezzo surfaces were proposed in [20]. The field theory for the cone over $\mathbf{d P}_{8}$ was also studied in [21], in relation to the fact that after a suitable symmetry breaking, it leads to a set of fields similar to the set of fields of the supersymmetric standard model.

2.2. The $\boldsymbol{R}$-symmetry and $\boldsymbol{a}$-maximization. An important check of the AdS/CFT correspondence in the case of toric del Pezzo surfaces $\left(\mathbf{d P}_{1}, \mathbf{d P}_{2}\right.$, and $\left.\mathbf{d} \mathbf{P}_{3}\right)$ is as follows. As mentioned above, the isometry group for the metrics on the cones over these surfaces includes at least $U(1)^{3}$. From the standpoint of the associated Sasaki-Einstein manifold, one of these $U(1)$ factors corresponds to the Reeb vector field, and from the gauge theory standpoint, it corresponds to the $R$-symmetry. The other two $U(1)$ factors (which arise from the $U(1) \times U(1)$ isometries of the del Pezzo surfaces themselves) are in fact additional flavor symmetries of the theory. In such theories, it is a complicated task to "separate" the global symmetries from the $R$-symmetry, i.e., to determine the charges of the elementary fields, for instance. The condition that the superpotential has a particular $R$-charge, as well as other physical considerations, are usually insufficient.

A systematic method for determining the $R$-charges of the theory based on certain relations between the triangle anomalies in $\mathcal{N}=1$ superconformal field theories was introduced in [22]. It consists in the following. It is known that in a general $d=4$ conformal field theory, the Weyl anomaly (i.e., the anomaly of the energy-momentum tensor) can be written as

$$
\left\langle T_{\mu}^{\mu}\right\rangle_{g}=a E_{4}+c W^{2}
$$

where $E_{4}$ is the Euler density and $W^{2}$ is the square of the Weyl tensor. What is meant here is that the trace of the energy-momentum tensor of a theory defined in the space with fixed background metric $g$ is computed in the left-hand side. Differentiating with respect to the metric and subsequently setting it to the flat metric, we can reformulate this equality: a correlation function of the elements of the energymomentum tensor with the insertion of the operator $T_{\mu}^{\mu}$ is nonzero. The quantities $a$ and $c$ (the central 
charges) are characteristic for the conformal field theory at hand and can be expressed in terms of the $R$-charges of elementary fields. In particular, we can calculate $a$ using the formula

$$
a=\frac{3}{32}\left(3 \operatorname{tr} R^{3}-\operatorname{tr} R\right)
$$

where tr denotes the sum over all fields of the theory. The prescription for finding the correct $R$-charges, introduced in [22], amounts to the following. All fields should be assigned arbitrary admissible $R$-charges (such that the superpotential would have the right dimensionality). We then find a local maximum of the function $a$ with respect to all the introduced parameters, i.e., with respect to all additional $U(1)$ factors, with which the $R$-charge can mix. As a result, we obtain a set of quadratic equations, whose solution gives the correct $R$-charges of all fields.

Knowing the $R$-charges is especially important because there is an alternative way to compute them, namely, using the AdS/CFT correspondence. The statement in [6], [23] is that the $R$-charges are in fact the volumes of special three-dimensional submanifolds of a given Sasaki-Einstein manifold $X^{5}$. It was shown in [24] that with the methods of toric geometry, calculating the volumes of such submanifolds is reducible to finding an extremum of a certain function, which hence provides a geometric counterpart to maximizing $a$.

\section{Nonconformal supersymmetric theories}

An important question is whether the AdS/CFT correspondence can be extended to the case of nonconformal field theories. For certain supersymmetric theories, the answer is affirmative. Two qualitatively different cases are the best studied: where the conformal symmetry is broken by condensation of scalar fields (i.e., via a Higgs effect) and where the original conformal model dual to $\operatorname{AdS}_{5} \times X^{5}$ is explicitly modified such that conformality is violated. We consider both cases.

3.1. Resolution of singularities and symmetry breaking. We return to the case of $\operatorname{AdS}_{5} \times S^{5}$ and the $\mathcal{N}=4 \mathrm{SYM}$. This field theory describes the low-energy fluctuations of a stack of $N$ coincident D3 branes in ten-dimensional space. Six scalar fields $\phi_{i}, i=1, \ldots, 6$, correspond to the six directions of normal brane fluctuations. The potential energy is minimum when $\left[\phi_{i}, \phi_{j}\right]=0$. We diagonalize these $N \times N$ matrices and assume that the eigenvalues split into two sets of $M$ and $N-M$ pairwise coinciding eigenvalues. From the field theory standpoint, the original $S U(N)$ symmetry is broken to $S(U(M) \times U(N-M))$. From the geometric standpoint, this means that two stacks of branes respectively containing $M$ and $N-M$ branes have been split from each other. This configuration can be explicitly described in the framework of supergravity. Indeed, we again seek the solution for the metric in form (4), but we do not require that the function $h$ depend only on the radial variable:

$$
d s^{2}=h^{-1 / 2}(\vec{r}) \sum_{i=1}^{4} d x_{i}^{2}+h^{1 / 2}(\vec{r})\left(d r^{2}+r^{2}(d \Omega)_{S^{5}}\right),
$$

where $\vec{r}$ are the coordinates of $\mathbb{R}^{6}$. It then follows from the equations of motion that

$$
\triangle_{\mathbb{R}^{6}} h=\text { sources }=\sum_{i} \delta\left(\vec{r}-\vec{r}_{i}\right),
$$

where "sources" are the positions of the D3 branes, which are pointlike objects from the standpoint of the transverse space $\mathbb{R}^{6}$.

The solution can be obtained by an elementary modification of $(5)$ ( $L$ is hereafter a linear scale):

$$
h=1+\sum_{i} \frac{L^{4}}{\left|\vec{r}-\vec{r}_{i}\right|^{4}} .
$$


It turns out that a substitution of type (10) is applicable in a much more general situation where the branes are placed at arbitrary points of the transverse Ricci-flat manifold $Y^{6}$. In what follows, we take the vicinity of a conical singularity of a Calabi-Yau variety as $Y^{6}$. Let $\left(d s^{2}\right)_{Y^{6}}$ be the metric on $Y^{6}$. Instead of (10), we can then write

$$
d s^{2}=h^{-1 / 2}(\vec{r}) \sum_{i=1}^{4} d x_{i}^{2}+h^{1 / 2}(\vec{r})\left(d s^{2}\right)_{Y^{6}},
$$

where $\vec{r}$ are the coordinates on $Y^{6}$. The equations of motion again lead to an equation of type (11), where we must take the Laplacian on $Y^{6}$ as a Laplacian and we still have a sum over the sources, i.e., over the positions of the D3 branes on $Y^{6}$ in the right-hand side. If $Y^{6}$ is a cone over the Sasaki-Einstein manifold $X^{5}$, then the displacements of the branes can be interpreted as nonzero vacuum expectation values of the fields in the dual (originally conformal) field theory [25]. But an important observation in [25] was that not every symmetry breaking in the dual gauge theory can be interpreted as a displacement of branes.

For instance, in the $\mathcal{N}=1$ theory with superpotential (9), we can consider classical vacuum configurations in which the only nonzero fields are the $A$ fields (or only the $B$ fields). In more invariant terms, we might say that the nonzero vacuum expectation value is acquired by a "baryonic" operator $\operatorname{det} A$ while the "mesonic" operators $\operatorname{tr}(A B)^{k}$ remain zero. The statement in [25] is that the nonzero expectation values of baryonic operators should be interpreted as a resolution of the singularity of the dual geometric cone. In the case of the conifold, because of the large group of isometries, the metric on the "resolved" space (i.e., the space with no singularity at the vertex) can be written explicitly [11]. A sphere $S^{2}$ in this space is glued in at the "point" $r=0$ (the vertex of the cone), and this "point" is therefore no longer singular.

Using ansatz (12) for the metric that takes the back-reaction of the D3 branes into account and again assuming that the function $h$ depends only on the radius, the authors of [12] found a solution $h(r)$ of the Laplace equation, but this solution turned out to be singular at $r=0$. This problem was resolved in [26], where it was shown that the presence of the singularity is a consequence of the ansatz in which $h$ is assumed to depend only on the radius. Physically, this means that the D3 branes are "smeared" over the sphere glued in at $r=0$. But if we assume that the branes are located in a single point $\vec{r}_{0}$ of the glued-in sphere, then we can find a smooth solution of the corresponding Laplace equation [26]. Moreover, although this solution asymptotically approaches $\operatorname{AdS}_{5} \times T^{11}$ as $r \rightarrow \infty$, the solution becomes $\operatorname{AdS}_{5} \times S^{5}$ in the vicinity of the branes, i.e., as $\vec{r} \rightarrow \vec{r}_{0}$. Such an interpolation can be interpreted as symmetry breaking in the original conformal theory by a baryonic condensate, which leads to $\mathcal{N}=4 \mathrm{SYM}$ in the infrared limit (i.e., after the massive fields are integrated out).

The cone over the rank-one del Pezzo surface. It is interesting to generalize this consideration to the case where the Calabi-Yau singularity is not (6) but a cone over a del Pezzo surface. We study the simplest case of the rank-one del Pezzo surface (the blow-up of $\mathbb{C P}^{2}$ at one point). Before solving for the Green's function $h$ that describes the gravitational back-reaction of the D3 branes placed on the cone, we must find a Ricci-flat metric $\left(d s^{2}\right)_{Y^{6}}$ on the cone itself. We can show that it should have the group of isometries $U(1) \times U(1) \times S U(2)$, i.e., in this case, we can seek a Kähler potential of the form [27]

$$
K=K\left(|u|^{2},\left|z_{1}\right|^{2}+\left|z_{2}\right|^{2}\right):=K\left(e^{t}, e^{s}\right) .
$$

It turns out to be useful to perform a Legendre transform with respect to $(s, t)$, introducing the dual variables

$$
\mu=\frac{\partial K}{\partial s}, \quad \nu=\frac{\partial K}{\partial t}
$$

and potential $G=\mu s+\nu t-K$. The Ricci-flatness equation then becomes

$$
e^{G_{\mu}+G_{\nu}}\left(G_{\mu \mu} G_{\nu \nu}-G_{\mu \nu}^{2}\right)=\mu .
$$


The variables $(\mu, \nu)$ are moment maps for the action of the group $U(1)^{2}$, and the function $G$ is defined on the corresponding moment polygon.

As $(\mu, \nu) \rightarrow \infty$, the metric should take the form of a cone $d s^{2}=d r^{2}+r^{2}\left(\widetilde{d s^{2}}\right)_{X^{5}}$ over some SasakiEinstein manifold $X^{5}$. In the variables $(\mu, \nu)$, this means that $G$ has the asymptotic form

$$
G_{0}=3 \nu(\log \nu-1)+\nu P_{0}(\xi) \quad \text { as } \mu, \nu \rightarrow \infty, \quad \xi=\frac{\mu}{\nu} .
$$

Substituting $G_{0}$ for $G$ in Eq. (13), we can find $P_{0}(\xi)$ as a solution of an ordinary differential equation. In fact, we can thus obtain the metrics of all the spaces $Y^{p, q}$ [15]. Formally, expression (14) provides an exact solution of Eq. (13), but this solution is singular at the cone vertex $r=0$ (i.e., for finite values of $\mu, \nu$ ). To resolve this singularity, we must seek corrections to $G_{0}$ in $1 / \nu$.

We seek the general solution in the form

$$
G=3 \nu(\log \nu-1)+\nu P_{0}(\xi)+\log \nu+\sum_{k=0}^{\infty} \nu^{-k} P_{k+1}(\xi)
$$

In the order $\nu^{-M}$, we obtain an ordinary differential equation for $P_{M}(\xi)$ :

$$
\frac{d}{d \xi}\left(Q(\xi) \frac{d P_{M}}{d \xi}\right)-\left((M-2)^{2}-1\right) \xi P_{M}=\text { right-hand side }
$$

where $Q(\xi)=\xi^{3}-3 \xi^{2} / 2+d$ and the right-hand side depends on the previous orders of the expansion, i.e., on $P_{0}, \ldots, P_{M-1}$. Equation (15) is a special case of a Heun equation: the Fuchsian equation with four regular singularities on the Riemann sphere. It was shown in [27] that the physical interval is $\xi \in\left[\xi_{1}, \xi_{2}\right]$, where $\xi_{1}$ and $\xi_{2}$ are the two largest roots of the polynomial $Q(\xi)$. It follows from the regularity requirement for the metric at $\xi=\xi_{1}, \xi_{2}$ that the function $P_{M}(\xi)$ must be real-analytic at those points, i.e., expandable in a Taylor series. Because $\xi=\xi_{1}, \xi_{2}$ are singularities of (15), a solution that is regular near both points, as a rule, does not exist; more precisely, it can only exist for particular values of the spectral parameter, which is equal to zero in (15).

We note that if the homogeneous equation

$$
\frac{d}{d \xi}\left(Q(\xi) \frac{d P_{M}}{d \xi}\right)-\left((M-2)^{2}-1\right) \xi P_{M}=0
$$

has a regular solution for some $M=1,2, \ldots$, then this solution can be taken with an arbitrary coefficient, which in turn should be interpreted as a new parameter of the metric on the cone. But it turns out that in the case of interest, regular solutions of (16) exist only for $M=3,4$ [27]. Therefore, the Ricci-flat metric on the cone over $\mathbf{d P}_{1}$ can have no more than two parameters. Furthermore, there is an additional topological relation between these parameters [28], and there is hence only one independent parameter of the metric remaining in the end. One exact metric on the cone over $\mathbf{d P}_{1}$ is known [28], [29], but the parameter in that metric is fixed, i.e., that metric is not the most general metric. What is the geometric meaning of this parameter? Our conjecture is that it corresponds to the size of the blown-up $\mathbb{C P}^{1}$ inside the rank-one del Pezzo surface. From the standpoint of the dual field theory constructed in [17], this parameter should correspond to a certain vacuum expectation value of one or several chiral fields (some of the symmetry-breaking patterns for this model were studied in [30]). It would be very interesting to elaborate this symmetry-breaking mechanism. 
3.2. Solutions with fractional branes. We return to the case of conifold (6). The conical Ricciflat metric on algebraic variety $(6), d s^{2}=d r^{2}+r^{2}\left(\widetilde{d s^{2}}\right)_{T^{11}}$, is singular at $r=0$. As discussed above (see Sec. 3.1), the singularity at the vertex of the cone can be removed using the blow-up of a sphere $\mathbb{C P}^{1}$. But this is not the only way to eliminate the singularity. The point is that the cone base $T^{11}$ is topologically a product $S^{2} \times S^{3}$, and we can blow up an $S^{3}$ instead of an $S^{2}$ at $r=0$. From the geometric standpoint, such a modification corresponds to "deforming" the conifold, namely, replacing Eq. (6) with the nonsingular equation

$$
X_{0}^{2}+X_{1}^{2}+X_{2}^{2}+X_{3}^{2}=\epsilon^{2} \neq 0 .
$$

A nonsingular Ricci-flat metric exists on such a "deformed" manifold [11]. Moreover, a related metric was presented in [31], but with a nonzero value of the field $F_{5}$, hence describing D3 branes embedded in this space. This metric has a singularity at $r=0$. There are also more general supergravity solutions related to the deformed conifold. One of them describes not only D3 branes but also D5 branes wrapped around the sphere $S^{2}$ in $T^{11}$ (these are also called "fractional" D3 branes) [32]. Such a solution does not have singularities, in contrast to the analogous solution on the unresolved (singular) conifold obtained previously [33]. The dual gauge theory has the gauge group $S U(N+M) \times S U(N)$ (here $M$ is the number of fractional branes). The change of the gauge group with respect to the case without fractional branes leads to an explicit breaking of conformal symmetry.

\section{Outlook}

Since its advent in 1997 and to the present, the AdS/CFT correspondence is one of the main directions of research in theoretical particle physics. The main quantitative confirmation of Maldacena's conjecture is related to studying the integrability of the $\mathcal{N}=4$ gauge theory [34] and the dual $\operatorname{AdS}_{5} \times S^{5}$ sigma model (see, e.g., [35]). Here, we have described several results from studying the AdS/CFT correspondence in the nonintegrable cases with a smaller amount of supersymmetry, mainly, in $\mathcal{N}=1$ theories. We showed that this case features Sasaki-Einstein manifolds and related positively curved complex surfaces. We are certain that understanding the geometric and physical aspects of these models will allow elucidating the more subtle aspects of the AdS/CFT correspondence and perhaps even the very physical reason for its existence. It is important to note that the AdS/CFT correspondence has not yet been explained from the "first principles" of quantum field theory nor classical gravity. Revealing the physical essence of the remarkable AdS/CFT conjecture remains one of the priorities of elementary particle physics.

Acknowledgments. It is a pleasure to take this opportunity to congratulate Andrei Alekseevich on his birthday, to wish him health, optimism, and enjoyment of life. The author also thanks him for the support always felt from him and hopes that it will be interesting for him to read about developments in gauge theory related to beautiful geometric constructions.

This work was supported in part by the Russian Foundation for Basic Research (Grant Nos. 14-0100695_a and 13-01-12405 ofi_m2) and the Program for Supporting Young Scientists and Candidates of Science (Grant No. MK-2510.2014.1).

\section{REFERENCES}

1. J. M. Maldacena, Adv. Theoret. Math. Phys., 2, 231-252 (1998); arXiv:hep-th/9711200v3 (1997).

2. M. R. Douglas and G. W. Moore, "D-branes, quivers, and ALE instantons," arXiv:hep-th/9603167v1 (1996).

3. S. Kachru and E. Silverstein, Phys. Rev. Lett., 80, 4855-4858 (1998); arXiv:hep-th/9802183v3 (1998).

4. J. M. Figueroa-O'Farrill, E. Hackett-Jones, and G. Moutsopoulos, Class. Q. Grav., 24, 3291-3308 (2007); arXiv:hep-th/0703192v4 (2007).

5. C. Bär, Commun. Math. Phys., 154, 509-521 (1993). 
6. D. Berenstein, C. P. Herzog, and I. R. Klebanov, JHEP, 0206, 047 (2002); arXiv:hep-th/0202150v2 (2002).

7. G. T. Horowitz and A. Strominger, Nucl. Phys. B, 360, 197-209 (1991).

8. D. R. Morrison and M. R. Plesser, Adv. Theoret. Math. Phys., 3, 1-81 (1999); arXiv:hep-th/9810201v3 (1998).

9. E. Calabi, Ann. Sci. École Norm. Sup. (4), 12, 269-294 (1979).

10. D. Bykov, "The Kähler metric of a blow-up," arXiv:1307.2816v1 [hep-th] (2013).

11. P. Candelas and X. C. de la Ossa, Nucl. Phys. B, 342, 246-268 (1990).

12. L. A. Pando Zayas and A. A. Tseytlin, Phys. Rev. D, 63, 086006 (2001); arXiv:hep-th/0101043v2 (2001).

13. I. R. Klebanov and E. Witten, Nucl. Phys. B, 536, 199-218 (1998); arXiv:hep-th/9807080v3 (1998).

14. A. Besse, Einstein Manifolds (Ergebn. Math. ihrer Grenz. (3), Vol. 10), Springer, Blin (2002).

15. J. P. Gauntlett, D. Martelli, J. Sparks, and D. Waldram, Adv. Theoret. Math. Phys., 8, $711-734$ (2004); arXiv:hep-th/0403002v3 (2004).

16. G. Tian and S.-T. Yau, Commun. Math. Phys., 112, 175-203 (1987).

17. B. Feng, A. Hanany, and Y.-H. He, Nucl. Phys. B, 595, 165-200 (2001); arXiv:hep-th/0003085v2 (2000).

18. S. Franco, A. Hanany, D. Martelli, J. Sparks, D. Vegh, and B. Wecht, JHEP, 0601, 128 (2006); arXiv:hep-th/ 0505211v3 (2005).

19. M. Cvetic, H. Lu, D. N. Page, and C. N. Pope, Phys. Rev. Lett., 95, 071101 (2005); arXiv:hep-th/0504225v4 (2005).

20. M. Wijnholt, Adv. Theoret. Math. Phys., 7, 1117-1153 (2004); arXiv:hep-th/0212021v3 (2002).

21. H. Verlinde and M. Wijnholt, JHEP, 0701, 106 (2007); arXiv:hep-th/0508089v2 (2005).

22. K. A. Intriligator and B. Wecht, Nucl. Phys. B, 667, 183-200 (2003); arXiv:hep-th/0304128v3 (2003).

23. E. Witten, JHEP, 9807, 006 (1998); arXiv:hep-th/9805112v1 (1998).

24. D. Martelli, J. Sparks, and S.-T. Yau, Commun. Math. Phys., 268, 39-65 (2006); arXiv:hep-th/0503183v3 (2005).

25. I. R. Klebanov and E. Witten, Nucl. Phys. B, 556, 89-114 (1999); arXiv:hep-th/9905104v2 (1999).

26. I. R. Klebanov and A. Murugan, JHEP, 0703, 042 (2007); arXiv:hep-th/0701064v2 (2007).

27. D. Bykov, "Comments on the del Pezzo cone," arXiv:1405.2319v1 [hep-th] (2014).

28. D. Martelli and J. Sparks, J. Geom. Phys., 59, 1175-1195 (2009); arXiv:0707.1674v2 [math.DG] (2007).

29. V. Apostolov, D. M. J. Calderbank, and P. Gauduchon, J. Differ. Geom., 73, 359-412 (2006).

30. D. Martelli and J. Sparks, JHEP, 0804, 067 (2008); arXiv:0709.2894v3 [hep-th] (2007).

31. L. A. Pando Zayas and A. A. Tseytlin, JHEP, 0011, 028 (2000); arXiv:hep-th/0010088v3 (2000).

32. I. R. Klebanov and M. J. Strassler, JHEP, 0008, 052 (2000); arXiv:hep-th/0007191v4 (2000).

33. I. R. Klebanov and A. A. Tseytlin, Nucl. Phys. B, 578, 123-138 (2000); arXiv:hep-th/0002159v2 (2000).

34. J. A. Minahan and K. Zarembo, JHEP, 0303, 013 (2003); arXiv:hep-th/0212208v3 (2002).

35. G. Arutyunov and S. Frolov, J. Phys. A, 42, 254003 (2009); arXiv:0901.4937v2 [hep-th] (2009). 